\title{
Algebraic Properties of the Index of Invariant Subspaces of Operators on Banach Spaces
}

\author{
GEORGE CHAILOS
}

\begin{abstract}
For an operator $S$ on a Banach space $X$, let Lat $(S, X)$ be the collection of all its invariant subspaces. We consider the index function on $\operatorname{Lat}(S, X)$ and establish various algebraic properties of it. Amongst others we show that if $S$ is a bounded below operator, then

$$
\text { ind } M+\operatorname{ind} N \geq \operatorname{ind}(M \cap N)+\operatorname{ind}(M \vee N) \text {. }
$$

If, in addition, ind $M=$ ind $N=1$ and $M \cap N \neq\{0\}$ then $\operatorname{ind}(M \vee N)=1$.
\end{abstract}

\section{Introduction}

If $S$ is an operator on a Banach space $X$, then a closed subspace $M$ of $X$ is called invariant for $S$ if $S M \subset M$. The collection of invariant subspaces of an operator $S$ is denoted by $\operatorname{Lat}(S, X)$. It forms a complete lattice with respect to intersections and closed spans. One of the important notions in the general theory of operators, such as bounded below operators, is the index of an element in $\operatorname{Lat}(S, X)$, which is defined as follows. (This definition is taken from [1].)

Definition 1.1. The map

$$
\text { ind }: \operatorname{Lat}(S, X) \longrightarrow\{0\} \cup \mathbb{N} \cup\{\infty\}
$$

is defined as ind $M=\operatorname{dim}(M / S M)$ and ind $M=0$ if and only if $M=\{0\}$. We say that $M$ has index $n$ if ind $M=n$.

The index function plays an essential role in the study of invariant subspaces of Banach spaces. (For example, see an extensive study in [5] of index 1 invariant subspaces in Banach spaces of analytic

2000 Mathematics Subject Classification. Primary: 47A15, 16D40. Secondary: 47A53.

Key words and phrases. Free modules, index, invariant subspaces. 
functions.) In this article we give various algebraic properties of the index function. Amongst others, and as a corollary to our main result, we show that if $M, N \in \operatorname{Lat}(S, X)$, ind $M=$ ind $N=1$ and $M \cap N \neq\{0\}$ then $\operatorname{ind}(M \vee N)=1$, where $M \vee N$ denotes the closed span of $M$ and $N$. (Equivalently, $M \vee N$ is the closure of $M+N$ ). This result, but in not such a general setting as the one presented here, was proved by Richter ([5], Corollary 3.12), using operator theoretical tools and results from analysis. Here we prove it using only algebraic tools and a rather standard result from functional analysis.

\section{Algebraic Properties of the Index Function}

Theorem 2.1. Let $\mathbf{R}$ be a commutative ring with identity and let $A, A^{\prime}, B^{\prime}$ be free unitary $\mathbf{R}$-modules such that $A^{\prime}$ and $B^{\prime}$ are free submodules of $A$. Then

$\operatorname{rank}\left(A / A^{\prime}\right)+\operatorname{rank}\left(A / B^{\prime}\right)=\operatorname{rank}\left(A /\left(A^{\prime} \cap B^{\prime}\right)\right)+\operatorname{rank}\left(A /\left(A^{\prime}+B^{\prime}\right)\right)$.

Proof. Consider the following sequence

$$
0 \longrightarrow A /\left(A^{\prime} \cap B^{\prime}\right) \stackrel{f}{\longrightarrow} A / A^{\prime} \oplus A / B^{\prime} \stackrel{g}{\longrightarrow} A /\left(A^{\prime}+B^{\prime}\right) \longrightarrow 0,
$$

where $f([y])=([y],[y]), g([x],[y])=[x-y]$ and $[\cdot]$ denotes the equivalence class in the appropriate quotient module. We claim that the sequence above is exact.

To prove the claim we first show that $f$ and $g$ are well-defined homomorphisms. Letting $[y] \in A /\left(A^{\prime} \cap B^{\prime}\right)$ and $x \in A^{\prime} \cap B^{\prime}$, we obtain that $f([y+x])=([y+x],[y+x])=([y],[y])$. Hence, $f$ is well defined. Moreover, $f$ is a homomorphism, since

$$
\begin{aligned}
f([y]+[z]) & =([y]+[z],[y]+[z])=([y],[y])+([z],[z]) \\
f(r[y]) & =(r[y], r[y])=r([y],[y]), r \in \mathbf{R} .
\end{aligned}
$$

Similarly, if $([x],[y]) \in A / A^{\prime} \oplus A / B^{\prime}$, and $x_{1} \in A^{\prime}, x_{2} \in B^{\prime}$, then

$$
\begin{aligned}
g\left(\left[x+x_{1}\right],\left[y+y_{1}\right]\right) & =\left[\left(x+x_{1}\right)-\left(y+y_{1}\right)\right] \\
& =\left[(x-y)+\left(x_{1}-y_{1}\right)\right]=[x-y],
\end{aligned}
$$

since $x_{1}-y_{1} \in A^{\prime}+B^{\prime}$. Thus, $g$ is well defined. 
Moreover, $g$ is a homomorphism, since

$$
\begin{aligned}
g\left(([x],[y])+\left(\left[x^{\prime}\right],\left[y^{\prime}\right]\right)\right) & =g\left([x]+\left[x^{\prime}\right],[y]+\left[y^{\prime}\right]\right) \\
& =g\left(\left[x+x^{\prime}\right],\left[y+y^{\prime}\right]\right) \\
& =\left[\left(x+x^{\prime}\right)-\left(y+y^{\prime}\right)\right]=\left[x-y+x^{\prime}-y^{\prime}\right] \\
& =[x-y]+\left[x^{\prime}-y^{\prime}\right]
\end{aligned}
$$

and

$$
g(r([x],[y]))=g([r x],[r y])=[r x-r y]=r[x-y], r \in \mathbf{R} .
$$

It remains to show that $\operatorname{ker} g=\operatorname{im} f$. For this let $([x],[y]) \in$ $A / A^{\prime} \oplus A / B^{\prime}$ be such that $\left.g([x]],[y]\right)=0$. Then $[x-y]=0$, and thus $x-y \in A^{\prime}+B^{\prime}$. This implies that $x+A^{\prime}=y+B^{\prime}$, i.e., $[x]_{A / A^{\prime}}=$ $[y]_{A / B^{\prime}}$ wherefore $\left([x]_{A / A^{\prime}},[y]_{A / B^{\prime}}\right) \in \operatorname{im} f$, and hence $\operatorname{ker} g \subset \operatorname{im} f$.

Conversely, if $([x],[y]) \in \operatorname{im} f$ then $x+A^{\prime}=y+B^{\prime}$ and hence $x+A^{\prime}+B^{\prime}=y+A^{\prime}+B^{\prime}$. It follows that $g([x],[y])=[x-y]=0$ so that $\operatorname{im} f \subset \operatorname{ker} g$. The proof of the claim is complete.

Since $A /\left(A^{\prime}+B^{\prime}\right)$ is a free module, it is in particular projective, and hence the above exact sequence splits (see [4]). Therefore

$$
A / A^{\prime} \oplus A / B^{\prime}=A /\left(A^{\prime} \cap B^{\prime}\right) \oplus A /\left(A^{\prime}+B^{\prime}\right) .
$$

This immediately implies that

$\operatorname{rank}\left(A / A^{\prime}\right)+\operatorname{rank}\left(A / B^{\prime}\right)=\operatorname{rank}\left(A /\left(A^{\prime} \cap B^{\prime}\right)\right)+\operatorname{rank}\left(A /\left(A^{\prime}+B^{\prime}\right)\right)$ concluding the proof of the theorem.

As every vector space is free over its ground field, the following is an immediate consequence of the above theorem.

Corollary 2.2. If $X$ is a Banach space and $S$ an operator on $X$, for all $M, N \in \operatorname{Lat}(S, X)$

$$
\text { ind } M+\operatorname{ind} N=\operatorname{ind}(M \cap N)+\operatorname{ind}(M+N) .
$$

In then case when $S$ is a bounded below operator, like the shift operator on Banach spaces of analytic functions, the following holds.

Lemma 2.3. Suppose $M, N \in \operatorname{Lat}(S, X)$, where $S$ is a bounded below operator on a Banach space $X$. Then

$$
\operatorname{ind}(M \vee N) \leq \operatorname{ind}(M+N) \leq \operatorname{ind} M+\operatorname{ind} N .
$$


Proof. If either ind $M$ or ind $N$ is infinite, then there is nothing to prove. So we may assume that ind $M<\infty$ and ind $N<\infty$. Thus there are finite-dimensional subspaces $M_{1}$ and $N_{1}$ of $M$ and $N$, respectively, such that $M=S M+M_{1}, \quad N=S N+N_{1}$, where $\operatorname{dim} M_{1}=i n d M$ and $\operatorname{dim} N_{1}=i n d N$. We find that

$$
\begin{aligned}
M+N & =S M+M_{1}+S N+N_{1} \\
& =S(M+N)+M_{1}+N_{1} \\
& \subseteq S(M \vee N)+\left(M_{1}+N_{1}\right) \\
& \subseteq M \vee N .
\end{aligned}
$$

Since $S$ is a bounded below operator, its range is closed (see, e.g., [2], Proposition 6.4, chapter VII), and hence the second to last expression is the sum of a closed and a finite-dimensional subspace, hence it is closed. Since $M+N$ is dense in $M \vee N$ we obtain that the last inclusion in above is actually an equality. From this it follows that

$\operatorname{ind}(M \vee N) \leq \operatorname{dim}\left(M_{1}+N_{1}\right)=\operatorname{ind}(M+N) \leq \operatorname{ind} M+\operatorname{ind} N$.

The next theorem, which is our main result, follows immediately from Corollary 2.2 and Lemma 2.3.

Theorem 2.4. If $X$ is a Banach space and $S$ a bounded below operator on $X$ then, for all $M, N \in \operatorname{Lat}(S, X)$,

$$
\operatorname{ind} M+\operatorname{ind} N \geq \operatorname{ind}(M \cap N)+\operatorname{ind}(M \vee N) \text {. }
$$

Corollary 2.5. Suppose that $M_{1}, M_{2} \in \operatorname{Lat}(S, X)$ are such that ind $M_{1}=$ ind $M_{2}=1$, where $S, X$ are as in the previous theorem. If $M_{1} \cap M_{2} \neq\{0\}$ then ind $\left(M_{1} \vee M_{2}\right)=1$.

Proof. If $M_{1} \cap M_{2} \neq\{0\}$ then $\operatorname{ind}\left(M_{1} \cap M_{2}\right) \geq 1$. As $\operatorname{ind}\left(M_{1} \vee M_{2}\right) \geq$ 1, Theorem 2.4 implies that ind $\left(M_{1} \vee M_{2}\right)=1$.

Example 2.6. In [5], Proposition (2.16 b), Richter considered the case where $S$ is the shift operator on any Banach space $\mathcal{B}$ of analytic functions on an open and connected subset of the complex plane. He showed that if $m \geq 2$ and there is a space in $\operatorname{Lat}(S, \mathcal{B})$ of index $m$, and furthermore if $n_{1}, n_{2} \in \mathbb{N} \cup\{\infty\}, n_{1}+n_{2}=m$, then there are invariant subspaces $N_{1}, N_{2}$ such that ind $N_{i}=n_{i}, i=1,2$ and $\operatorname{ind}\left(N_{1} \vee N_{2}\right)=i n d N_{1}+i n d N_{2}$. In these cases, Theorem 2.4 implies that $\operatorname{ind}\left(N_{1} \cap N_{2}\right)=0$ and hence $N_{1} \cap N_{2}=\{0\}$. Thus, $N_{1} \vee N_{2}=$ 
$N_{1} \oplus N_{2}$. (For example, it is well known ([3], Corollary 6.5) that when $S$ is the shift operator on a weighted Bergman space on the unit disk, then for all $1 \leq m \leq \infty$ there are invariant subspaces of index $m$.)

\section{REFERENCES}

[1] G. Chailos, On Reproducing Kernels and Invariant Subspaces of the Bergman Shift, Ph.D. dissertation, University of Tennessee, Knoxville, 2002.

[2] J.B. Conway, A Course in Functionl Analysis, 2nd ed., Springer-Verlag, New York, 1990.

[3] H. Hedenmalm, B. Korenblum and K. Zhu, Theory of Bergman Spaces, Springer-Verlag, New York, 2000.

[4] T. Hungerford, Algebra, Springer-Verlag, New York, 1996.

[5] S. Richter, Invariant Subspaces in Banach Spaces of Analytic Functions, Trans. Amer. Math. Soc. 304 no.2 (1987), 585-616.

George Chailos,

Department of Computer Science,

University of Nicosia,

Nicosia 1700, Cyprus,

chailos.g@unic.ac.cy

Received on 6 June 2008. 